\title{
Extreme Events Characterization on Time Series
}

\author{
Marcos Wander Rodrigues, Luis Enrique Zárate \\ Pontifical Catholic University of Minas Gerais, Brazil \\ marcoswanderrodrigues@gmail.com, zarate@pucminas.br
}

\begin{abstract}
The use of sensors in environments where they require constant monitoring has been increasing in recent years. The main goal is to guarantee the effectiveness, safety, and smooth functioning of the system. To identify the occurrence of abnormal events, we propose a methodology that aims to detect patterns that can lead to abrupt changes in the behavior of the sensor signals. To achieve this objective, we provide a strategy to characterize the time series, and we use a clustering technique to analyze the temporal evolution of the sensor system. To validate our methodology, we propose the clusters' stability index by windowing. Also, we have developed a parameterizable time series generator, which allows us to represent different operational scenarios for a sensor system where extreme anomalies may arise.
\end{abstract}

CCS Concepts: • Applied computing;

Keywords: Anomaly Detection, Characterization of Time Series, Cluster Analysis, Extreme Events, Time Series Analysis

\section{INTRODUÇÃO}

Ambientes industriais críticos como barragens, sistemas de alto forno, sistemas de fornecimento de energia, gasodutos, e outros, possuem monitoramento contínuo baseado em sensores para garantir a integridade e a segurança, evitando desastres com vítimas, prejuízos econômicos e/ou ambientais.

De acordo com os dados temporais provenientes de sensores, o monitoramento pode apontar três possíveis cenários: a) predomínio da normalidade com algum nível de tendência; b) Variações abruptas que devem ser observadas e analisadas; e c) ocorrência de eventos críticos anômalos ou extremos, os quais devem receber maior atenção.

Para sistemas de monitoramento por sensores, a análise por séries temporais é a modelagem ideal para compreender os padrões de comportamento, prever anomalias e níveis de criticidade. Detectar e caracterizar esses níveis, ainda que não sejam eventos raros (com mínima probabilidade de ocorrer e com maior impacto) [Taleb 2007], permitiria a construção de sistemas de alerta mais eficientes.

A partir dos registros dos sistemas de sensores é possível caracterizar as séries temporais extraindo atributos como valor máximo, mínimo, média, tendência, desvio padrão, além de outras características que podem representar uma série temporal [Lee et al. 2014]. Após caracterização por janelas temporais, é possível aplicar técnicas de agrupamento para reconhecer e prever padrões que melhor descrevam a evolução temporal de determinados eventos.

Em sistemas reais de ambientes críticos, dados de monitoramento, provenientes dos sensores, não são normalmente disponibilizados. Por esse motivo, e para este trabalho optamos por construir um gerador de sinais (séries temporais), o qual permite a geração de bases de dados temporais sintéticas para representar diferentes condições de operação de sensores.

Neste artigo, são extraídas características do conjunto de séries temporais (sinais de sensores) gera-

À CAPES - Coordenação de Aperfeiçoamento de Pessoal de Nível Superior - Código 001, CNPq - Conselho Nacional de Desenvolvimento Científico e Tecnológico e FAPEMIG - Fundação de Amparo à Pesquisa do Estado de Minas Gerais. Copyright(C)2020 Permission to copy without fee all or part of the material printed in KDMiLe is granted provided that the copies are not made or distributed for commercial advantage, and that notice is given that copying is by permission of the Sociedade Brasileira de Computação. 
dos sinteticamente, para posterior aplicação de técnicas de agrupamento, a fim de caracterizar anomalias que possam ocorrer de modo a relacioná-las ao surgimento de eventos extremos. Este trabalho, faz parte de um projeto maior que procura observar os movimentos por janelamento, nas respostas dos sensores, extraindo padrões dessas mudanças operacionais.

A contribuição principal deste trabalho é a proposta de um procedimento para analisar a evolução temporal de um sistema de sensores envolvendo condições normais sujeitas à variações abruptas e à variações anômalas de operação.

O artigo possui a seguinte organização: A Seção 2 descreve brevemente os trabalhos relacionados. Na Seção 3 a fundamentação teórica que sustenta nosso trabalho é descrita. Na Seção 4 a metodologia adotada e uma breve descrição do gerador de séries temporais é descrito. Na Seção 5 os experimentos e análise dos resultados são apresentados. Finalmente, a Seção 6 conclui e expõe os trabalhos futuros.

\section{TRABALHOS RELACIONADOS}

A análise de séries temporais tem um papel fundamental na predição de novos eventos dado os eventos que ocorreram no passado. Seja no contexto uni-variado ou multivariado, é possível agrupar e classificar as séries temporais a fim de realizar predições em cenários dinâmicos devido ao fator temporal.

Os diversos contextos que utilizam as séries temporais demandam diferentes estratégias de análises. O trabalho de [Yanfei Kang 2018] propõe o uso de modelos mixture autoregressive para identificar a similaridade entre séries temporais. Em [Abdullah 2014], os autores usam as representações como a transformada discreta de Fourier, medidas de similaridade por correlação, e a transformada discreta de wavelets para caracterizar a segmentação de séries temporais em diversas aplicações de Motifs.

Considerando a ocorrência de valores extremos, o trabalho de [Chavez-Demoulin and Davison 2012] utiliza da análise uni-variada em séries temporais para identificar o surgimento de anomalias nos valores da série. Enquanto que na análise multivariada, o trabalho de [Ranjan et al. 2018] busca identificar um conjunto específico de variáveis que podem ser responsáveis na identificação de um possível evento extremo. O objetivo dos autores é prever o surgimento desses eventos por meio de modelos supervisionados de machine-learning (ML).

Algoritmos de agrupamento são técnicas de ML utilizadas para investigar o comportamento de uma série temporal específica dentro dos clusters [Nakkeeran et al. 2012]. Assim, os autores buscam extrair informação relevante destes clusters a fim de melhorar a eficácia de sistemas decisórios. O trabalho de [Serra and Zárate 2015] também utiliza as técnicas de agrupamento para tentar descrever o comportamento de séries temporais em um conjunto de clusters, bem como o surgimento de novos clusters, a partir de características como a componente de nível e tendência extraídas da série.

A provável ocorrência de eventos com valores anômalos na série temporal, torna o seu tratamento e análise inadequados por meio da distribuição gaussiana. Sendo, assim, necessário o uso de métodos estatísticos como a teoria de valores extremos (TVE) para lidar com tais anomalias [Caires 2011].

\section{FUNDAMENTAÇÃO TEÓRICA}

\subsection{Séries Temporais}

Uma série temporal (ST) é uma sequência temporal de dados discretos, e um conjunto de STs compõe uma base de dados temporais (TDB). As STs regulares são geradas em intervalos de tempo regularmente espaçados, já as STs irregulares são geradas em intervalos de tempo variados. A TDB gerada para este trabalho é multivariada e formada por sinais de sensores (STs regulares) expressos pela

Symposium on Knowledge Discovery, Mining and Learning, KDMILE 2020 - Applications Track. 
matriz da Equação 1.

$$
[Z]=\left[\begin{array}{cccc}
Z_{t^{11}} & Z_{t^{12}} & \cdots & Z_{t^{1 M}} \\
Z_{t^{21}} & Z_{t^{22}} & \cdots & Z_{t^{2 M}} \\
\vdots & \vdots & \ddots & \vdots \\
Z_{t^{N 1}} & Z_{t^{N 2}} & \cdots & Z_{t^{N M}}
\end{array}\right]_{N \times M}
$$

onde cada elemento $Z_{t^{i j}}=\left\{Z_{1^{i j}}, Z_{2^{i j}}, \cdots, Z_{T^{i j}}\right\}$, representa uma série temporal gerada pelo sensor $(i, j)$ para $i=\{1, \cdots, N\}$ e $j=\{1, \cdots, M\}$, onde $N$ representa o número de arrays de sensores, e $M$ o número de sensores por array. O elemento $Z_{t^{i j}}$ corresponde a uma observação para o sensor $j$ no array $i$, e cada valor de $t=\{1, \cdots, T\}$ corresponde aos períodos de observação na série. O número total de observações de $|Z|$ na TDB é dado por $|Z|=N \times M \times T$.

Uma ST é representada por modelos que podem variar de acordo com os objetivos da análise, podendo ser modelos não-paramétricos ou paramétricos. A representação deste último considera a forma geral de uma ST dada pela Equação $Z_{t}=f_{t}+a_{t}$, onde $f_{t}$ é uma função do tempo somada ao ruído $a_{t}$. Neste artigo, usamos o modelo de suavização de Holt-Winters [Winters 1960], o qual utiliza as componentes de nível $\left(\mu_{t}\right)$, tendência $\left(T_{t}\right)$, sazonalidade $\left(F_{t}\right)$, e o ruído $\left(a_{t}\right)$ associados a cada ST. Em STs geradas por um sistemas de sensores, a componente sazonalidade $\left(F_{t}\right)$ pode não ser considerada, uma vez que o processo de caracterização das séries ocorrem em curtos períodos de tempo, ou seja, são definidas pelas amplitudes das janelas $w_{h}$. Assim, o modelo utilizado neste trabalho é definido pela Equação $Z_{t}=\mu_{t}+T_{t}+a_{t}$.

\subsection{Janelamento e Caracterização de Séries Temporais}

Neste trabalho, consideramos que cada ST, $\left(Z_{t^{i j}}\right)$, é dividida em $H$ janelas definidas como $W=$ $\left\{w_{1}, w_{2}, \cdots, w_{H}\right\}$. Alguns autores têm discutido diversas estratégias para o janelamento de STs, como pode ser visto em [Huang et al. 2012].

O nosso objetivo é definir janelamentos apropriados para análise, ou seja, onde mudanças extremas podem ocorrer. Assim, o conhecimento acerca do sistema onde as STs são coletadas, nos permite conjecturar a periodicidade das variações, e consequentemente, a amplitude ideal do janelamento. Como critério de escolha da amplitude da janela $w_{h}$, devem ser observadas as séries que possuem menor variabilidade no tempo [Serra and Zárate 2015]. Para este trabalho consideramos janelas com tamanho fixo de 6 pontos discretos. As duas primeiras janelas $w_{1}$ e $w_{2}$ são utilizadas para geração de modelos de referência para efeito de comparação das possíveis movimentações dos sensores.

Como os dados gerados por uma ST variam continuamente no tempo, é relevante utilizar novos atributos que representem melhor todo o conjunto de STs. Como mencionado, o modelo clássico de análise de ST considera a média, tendência e sazonalidade. No entanto, outras técnicas de extração de características podem ser aplicadas para extração de características [Trovero and Leonard 2018]. Neste trabalho, consideramos cinco (5) componentes para cada série $Z_{t^{i j}}$ :

1) Mínimo: $\bar{M}_{i j h}$ é o valor mínimo da ST $(i, j)$ na janela $h$ de tempo;

2) Máximo: $\bar{X}_{i j h}$ é o valor máximo da ST $(i, j)$ na janela $h$ de tempo;

3) Média: $\bar{Z}_{i j h}$ é a média de nível da ST $(i, j)$ na janela $h$ de tempo;

4) Inclinação: $\hat{T}_{i j h}$ é a tendência da ST $(i, j)$ na janela $h$ de tempo;

5) Desvio padrão: $\bar{S}_{i j h}$ é a medida de dispersão da ST $(i, j)$ na janela $h$ de tempo.

A matriz $Z^{*}(2)$ contem os vetores de características $Z_{i j h}^{*}$ composto pelas componentes citadas anteriormente, $Z_{i j h}^{*}=\left[\bar{M}_{i j h}, \bar{X}_{i j h}, \bar{Z}_{i j h}, \hat{T}_{i j h}, \bar{S}_{i j h}\right]$. A cardinalidade da matriz [Z*] corresponde à 
$\left[Z^{*}\right]=N \times M \times H \times 5$.

$$
\left[Z^{*}\right]=\left[\begin{array}{cccc}
Z_{11}^{*} & Z_{121}^{*} & \cdots & Z_{1 M 1}^{*} \\
Z_{211}^{*} & Z_{221}^{*} & \cdots & Z_{2 M 1}^{*} \\
\vdots & \vdots & \ddots & \vdots \\
Z_{N 11}^{*} & Z_{N 21}^{*} & \cdots & Z_{N M 1}^{*} \\
\cdots & \cdots & \cdots & \cdots \\
Z_{12}^{*} & Z_{122}^{*} & \cdots & Z_{1 M 2}^{*} \\
Z_{212}^{*} & Z_{222}^{*} & \cdots & Z_{2 M 2}^{*} \\
\vdots & \vdots & \ddots & \vdots \\
Z_{N 12}^{*} & Z_{N 22}^{*} & \cdots & Z_{N M 2}^{*} \\
\cdots & \cdots & \cdots & \cdots \\
\ddots & \ddots & \ddots & \ddots \\
\cdots & \cdots & \cdots & \cdots \\
Z_{11 H}^{*} & Z_{12 H}^{*} & \cdots & Z_{1 M H}^{*} \\
Z_{21 H}^{*} & Z_{22 H}^{*} & \cdots & Z_{2 M H}^{*} \\
\vdots & \vdots & \ddots & \vdots \\
Z_{N 1 H}^{*} & Z_{N 2 H}^{*} & \cdots & Z_{N M H}^{*}
\end{array}\right]_{N \times M \times H}
$$

\section{METODOLOGIA}

A metodologia adotada é ilustrada na Fig. 1. Esta tem início com a parametrização do gerador de séries temporais SyTSBox, desenvolvido para este trabalho, e que permitiu gerar uma TDB para representar a operação de $S$ sensores em ambientes industrias ou de IoT. A segunda fase consiste no janelamento temporal $\left(w_{h}\right)$ da TDB. Na terceira fase da metodologia, é realizada a extração de características a fim de representar o conjunto de sensores $s_{j} \in S$. Na quarta fase, aplicamos algoritmo de clusterização Aglomerativa (AGNES) para analisar a movimentação dos sensores. Por fim, a quinta fase, analisamos o surgimento de eventos considerados anômalos ou extremos.

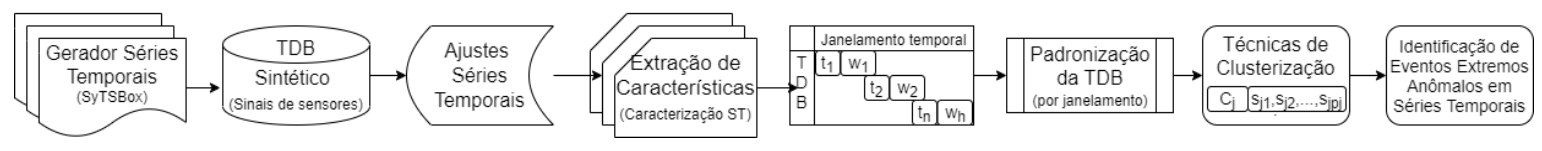

Fig. 1. Esboço da metodologia proposta

\subsection{Base de dados Temporal}

Devido à indisponibilidade de dados de STs em ambientes críticos, desenvolvemos um gerador de séries temporais capaz de emular diversos tipos de STs, gerando assim uma TDB sintética. O gerador, denominado de SyTSBox (Synthetic Time Series Box), é capaz de representar diferentes cenários de streaming devido às diversas configurações possíveis, dada por sua capacidade parametrizável.

Para este trabalho, a TDB gerada pelo SyTSBox é composta por 4 tipos de STs: 1) STs com indicador de tendência; 2) STs com ocorrência de picos; 3) STs com deslocamento abrupto de nível; e 4) STs com deslocamento gradual de nível. Exemplos dos tipos de STs são ilustradas nas Fig. 2, 3, 4 e 5. As STs que compõem a TDB são formadas por 40 sensores operando individualmente, onde cada sensor possui 240 pontos/instâncias de série. Os sensores foram definidos com os seguintes parâmetros operacionais: a) média $(\mu)$ aleatória entre 1 e 10 por sensor; b) desvio padrão $(\sigma)$ igual a $1 ;$ c) desvio padrão $(\sigma)$ entre 1 e 6 ; e d) cada sensor possui $5 \%$ de pontos com valores extremos. 


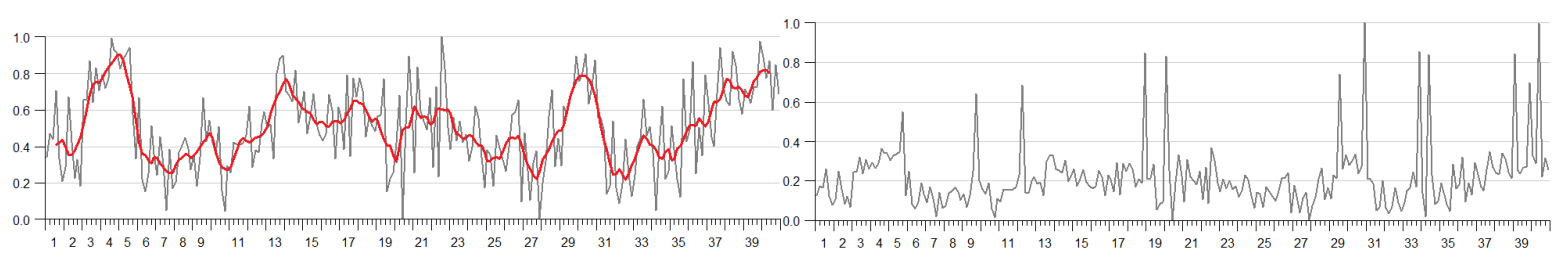

Fig. 2. ST com tendências

Fig. 3. ST com ocorrência de picos

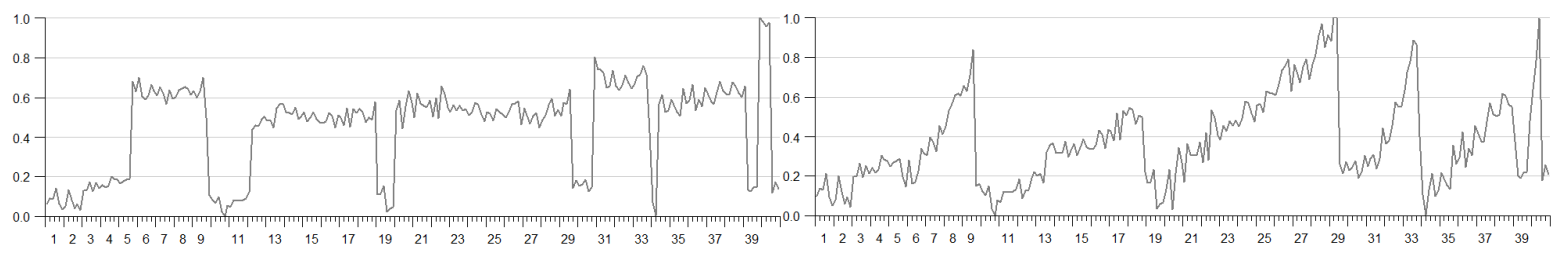

Fig. 4. ST com deslocamentos abruptos de média Fig. 5. ST com deslocamentos gradual de média

A Fig. 6 ilustra a variação dos sinais que compõem a TDB, tal como a presença de outliers nas STs com ocorrência de picos (VP1 VP10), e nas STs com deslocamento gradual de nível (VSG1 VSG10). No entanto, existe menor distância dos máximos em relação ao $3^{\circ}$ quartil nas STs com tendência (VT1 VT10), e maior distância nas STs com deslocamento abrupto de nível (VSA1 VSA10).

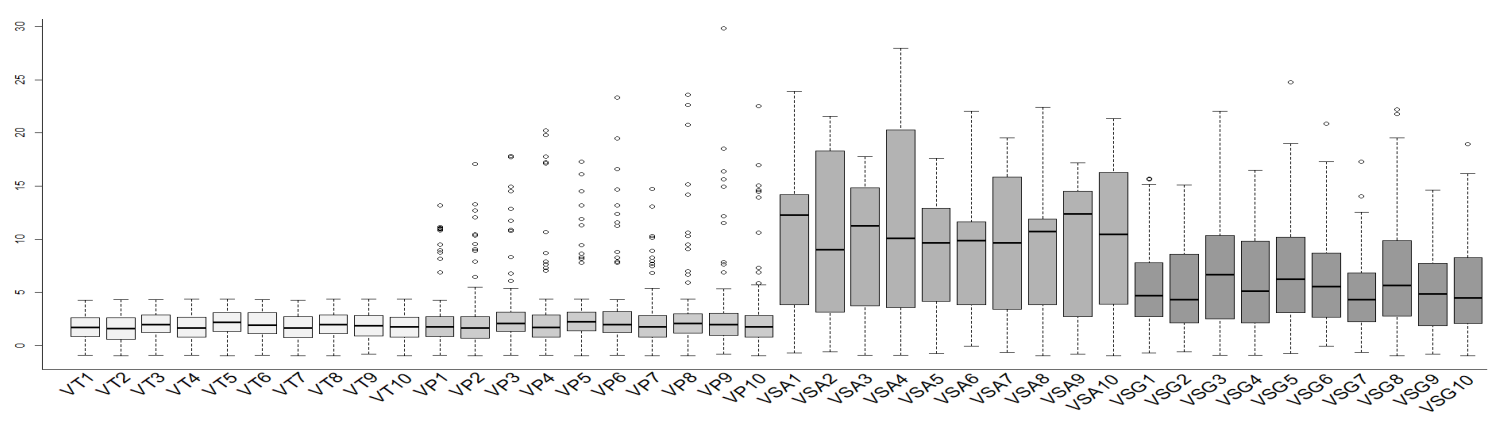

Fig. 6. Distribuição e valores dos sinais do sistema de sensores

\subsection{Tipos de Cenários}

Seja $s_{j}$ um sensor do conjunto $S$ de sensores e $K$ o número de clusters obtidos sob condições normais de operação. $C_{j}$ representa um determinado cluster definido por $C_{j}=\left\{s_{j 1}, \cdots, s_{j p_{j}}\right\}, \forall s_{j} \subseteq S, j=$ $\{1, \cdots, K\}$, onde, $p_{j}$ é o número de sensores no cluster $C_{j}$. De acordo com a evolução temporal nos sinais dos sensores, consideramos dois possíveis cenários, são eles:

Cenário I - Condições normais de operação:

O número de clusters é constante ao longo do tempo:

$$
\left|C_{j}^{\text {Referencia }}\right|=\left|C_{j}^{t}\right|=K
$$

Os sensores podem ou não mudar de agrupamentos:

$$
\left\{\begin{array}{l}
C_{j}^{\text {Referencia }}=C_{j}^{t} \\
C_{j}^{\text {Referencia }} \neq C_{j}^{t}
\end{array} \forall j=\{1, \cdots, K\}, t=\{1, \cdots, T\}\right.
$$


Cenário II - Existe variação significativa (eventos anômalos) nos sinais dos sensores: Número de clusters pode variar:

$$
\left|C_{j}^{\text {Referencia }}\right| \neq\left|C_{j}^{t}\right|
$$

\subsection{Clusterização das Séries Temporais}

A clusterização é feita sobre o conjunto de características $Z_{i j h}^{*}$ extraídas da janela $w_{h} \in W$ pertencentes aos sensores $s_{i} \in S$ da TDB. O número ótimo de clusters $C_{j}$ é obtido pela medida do Silhouette index. As estratégias para a clusterização das STs que atendem aos cenários $I$ e II são:

- Estratégia 1 (Cenário I) - O Silhouette index permite determinar o número ótimo de clusters para as janelas de referência $w_{1}$ e $w_{2}$, usando esse mesmo número para as janelas seguintes. Sendo o número de clusters constante (Equação 3), é possível verificar o índice de Estabilidade (Equação 6 ) dos sensores, o qual indica o grau de movimentação dos sensores entre os clusters.

- Estratégia 2 (Cenário II) - Por meio do Silhouette index é determinado o número ótimo de clusters para cada janela $w_{h} \in W$, onde $h=\{3, \cdots, 40\}$. Como o número de clusters pode ser variável (Equações 5), verifica-se o surgimento, ou o desaparecimento de clusters, o que indica alterações nas condições normais de operação de um ou mais sensores no sistema.

\subsection{Métricas de Avaliação}

4.4.1 Índice de Estabilidade. O indice Estabilidade $B\left(w_{h}\right)$ mede o quanto os sensores apresentamse estáveis nos clusters ao longo das janelas. $B\left(w_{h}\right)$ é a taxa de permanência do sensor no cluster representativo durante as janelas $w_{h}$, em relação aos modelos de referência, ver Equação 6 .

$$
B\left(w_{j}\right)=\frac{\mid \text { cluster }: w_{[1,2]} \cap \text { clusters }: w_{h} \mid}{\mid \text { clusters }: w_{[1,2]}|+| \text { clusters }: w_{h} \mid}
$$

onde, obtemos uma taxa de acerto ao comparar cada janela $w_{h}$ com os modelos de referência $w_{[1,2]}$.

4.4.2 Índice de Instabilidade. O indice Instabilidade $L\left(s_{i}\right)$ mede o quanto os sensores movimentamse entre os clusters. Assim, definimos a instabilidade (entropia) como, o quanto que cada sensor $s_{i}$ movimenta-se entre diferentes clusters nas $w_{j}$ janelas, ver Equação 7.

$$
L\left(s_{i}\right)=-\sum_{s \in S} P\left(s_{i}\right) \log _{2}\left(P\left(s_{i}\right)\right)
$$

O cálculo da instabilidade não utiliza os modelos de referência $\left(w_{[1,2]}\right)$, apenas a variação de $s_{i} \mathrm{em} w_{j}$.

\section{EXPERIMENTOS E ANÁLISE DOS RESULTADOS}

Considerando a Estratégia 1 (Seção 4.3), a medida Silhouette index indicou o número ideal de 2 clusters para ambos os modelos de referência, $w_{1}$ e $w_{2}$. Os clusters foram avaliados pelo teste de hipóteses multivariado $T^{2}$ de Hotelling [Hotelling 1992].

A divisão dos sensores nos clusters evidenciou a separação do conjunto de STs com tendência (em condições normais de operação) no cluster 1 (VT1 VT10); e em relação ao cluster 2 composto pelas séries com picos (VP1 VP10), séries com deslocamentos abruptos (VSA1 VSA10) e deslocamentos graduais (VSG1 VSG10), os quais apresentam valores anômalos, discrepantes das séries com condições normais de operação.

Pela Estratégia 1, foi aplicada o algoritmo de clusterização a cada janela $w_{1 . .40} \subseteq W$, individualmente (considerando a Equação 3). Assim, os sensores localizados nos clusters de cada janelamento 
$\left(w_{[3.40]}\right)$ são comparados com os clusters do modelo de referência $\left(w_{[1,2]}\right)$. Para avaliar o equilíbrio dos sensores nos clusters foi calculado o índice de Estabilidade (Equação 6) ilustrado na Tabela I.

\begin{tabular}{rll}
\hline Wnd & Clst & $\begin{array}{l}\text { Estabilidade } \\
\text { Ref[1,2] }\end{array}$ \\
\hline W1 & 2 & 1.000 \\
W2 & 2 & 1.000 \\
W3 & 2 & 0.975 \\
W4 & 2 & 1.000 \\
W5 & 2 & 0.625 \\
W6 & 2 & 0.225 \\
W7 & 2 & 0.275 \\
W8 & 2 & 0.500 \\
W9 & 2 & 0.600 \\
W10 & 2 & 0.025 \\
\hline
\end{tabular}

Table I. Índice de Estabilidade por janelamento

\begin{tabular}{|c|c|c|c|c|c|c|c|c|}
\hline Wnd & Clst & $\begin{array}{l}\text { Estabilidade } \\
\text { Ref[1,2] }\end{array}$ & Wnd & Clst & $\begin{array}{l}\text { Estabilidade } \\
\text { Ref[1,2] }\end{array}$ & Wnd & Clst & $\begin{array}{l}\text { Estabilidade } \\
\text { Ref[1,2] }\end{array}$ \\
\hline W11 & 2 & 1.000 & W21 & 2 & 0.650 & W31 & 2 & 0.325 \\
\hline W12 & 2 & 0.625 & $\mathrm{~W} 22$ & 2 & 0.725 & W32 & 2 & 0.475 \\
\hline W13 & 2 & 0.725 & W23 & 2 & 0.275 & W33 & 2 & 0.400 \\
\hline W14 & 2 & 0.700 & W24 & 2 & 0.525 & W34 & 2 & 0.275 \\
\hline W15 & 2 & 0.500 & W25 & 2 & 0.475 & W35 & 2 & 0.725 \\
\hline W16 & 2 & 0.500 & W26 & 2 & 0.500 & W36 & 2 & 0.400 \\
\hline W17 & 2 & 0.525 & W27 & 2 & 0.575 & W37 & 2 & 0.500 \\
\hline W18 & 2 & 0.450 & W28 & 2 & 0.525 & W38 & 2 & 0.525 \\
\hline W19 & 2 & 1.000 & W29 & 2 & 0.675 & W39 & 2 & 0.675 \\
\hline W20 & 2 & 0.600 & W30 & 2 & 0.925 & W40 & 2 & 0.650 \\
\hline
\end{tabular}

Considerando a Estratégia 2 (Seção 4.3), o número ideal de clusters foi obtido para cada janelamento $w_{1 . .40}^{6} \subseteq W$ Por este motivo, obtivemos um número diferente de clusters a cada janelamento $w_{j}$.

Para avaliar a movimentação dos sensores entre os clusters em cada janelamento, foi calculado o índice de Instabilidade (Equação 7), tanto para Estratégia 1 quanto para a Estratégia 2. Por meio do índice de Instabilidade foi possível identificar os sensores com mais instabilidades, ou seja, os sensores que tiveram maior variação nos valores de seus sinais, ver Tabela II.

Table II. Índice de Instabilidade por sensor com número fixo e variável de clusters

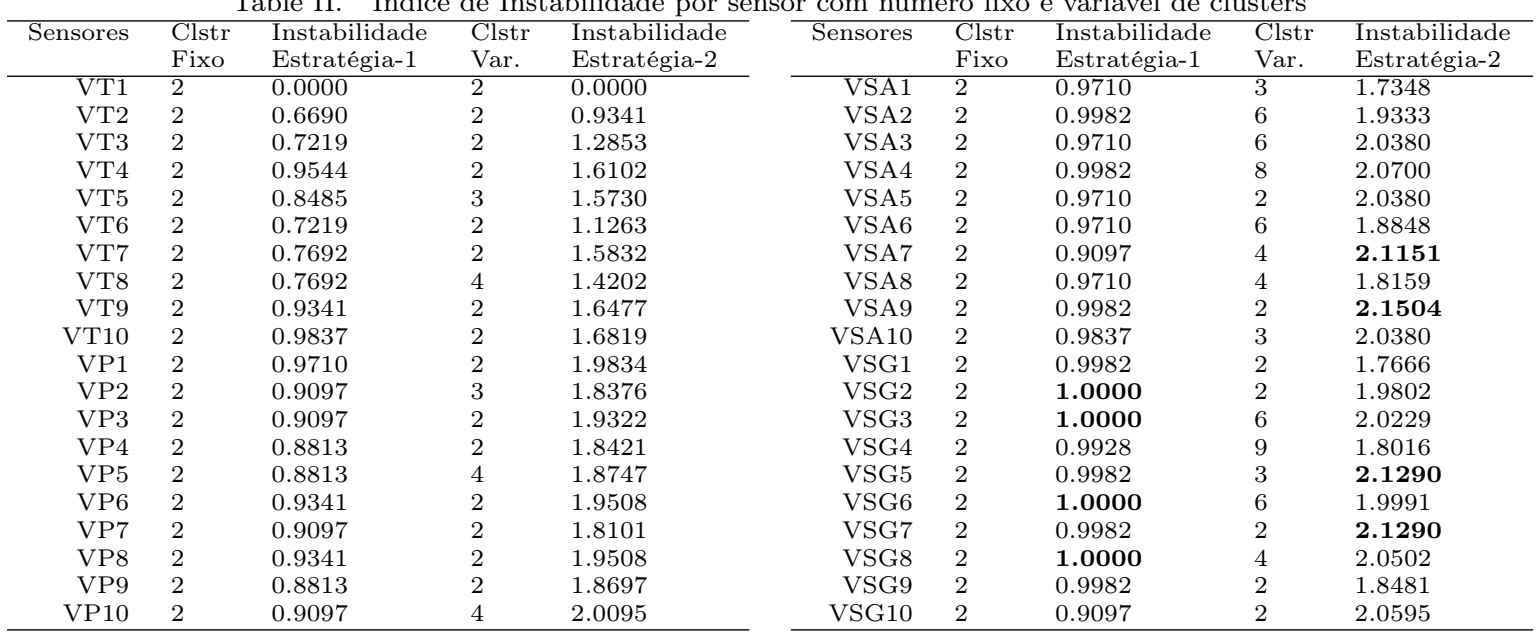

Note que, por meio do índice de Instabilidade, foi possível identificar os sensores que se mostraram mais instáveis, obtendo os valores de 1.0 e 2.1 para as Estratégias 1 e Estratégia 2, respectivamente.

Como mencionado, os sensores que compõem o cluster 1 contêm apenas as séries com indicadores de tendência (Fig. 2), não sendo possível identificar valores anômalos, ou mesmo indícios de algum valor extremo. Assim, os sensores contidos neste cluster não estão associados a eventos extremos. Porém, o cluster 2 é composto por séries que apresentam, além do comportamento anômalo, como picos (Fig. 3), deslocamento abrupto de médias (Fig. 4), e deslocamento gradual de médias (Fig. 5 ), também possui sinais com valores extremos, tornando o sistema de sensores que compõem este cluster, ideal para serem monitorados pela Teoria de Valores Extremos (Leonard Tippett 1902-1985). $\mathrm{O}$ índice de estabilidade 1.0 indica que não houve mudanças dos sensores entre os clusters. Valor menores no índice, indica movimentação dos sensores entre os clusters. 


\section{CONCLUSÃO}

Neste trabalho, abordamos o problema de análise em séries temporais que representam diferentes sistemas de sensores, onde a criticidade em termos de segurança deve ser monitorada. A estratégia de janelamento temporal foi efetiva, uma vez que o tamanho da janela foi definido pela menor variação da série. Esta efetividade foi demonstrada ao monitorar as demais janelas em relação aos modelos de referência, sendo possível identificar as mudanças de comportamento dos sensores entre clusters.

Como pontos a serem melhorados em nossa abordagem, apontamos: a) utilizar outras características para representar as séries temporais; b) construir modelos supervisionados para explicar as mudanças detectadas; c) considerar outros ajustes no tamanho do janelamento; d) utilizar um threshold semisupervisionado para diferenciar anomalias comuns dos grandes desvios; e) analisar o comportamento e a evolução temporal; e f) considerar a evolução dos modelos de referência pelas mudanças nos sensores.

Como trabalhos futuros, considera-se: implementar novas funções ao gerador SyTSBox, considerando outros cenários de séries temporais; definir um threshold que diferencie valores anômalos e extremos; guardar estados intermediários para ajustar o modelo de referência; e utilizar a teoria de valores extremos, como gatilho para detecção de eventos anômalos ou extremos em séries temporais.

\section{ACKNOWLEDGMENT}

The authors acknowledge the financial support from the CNPq (Brazilian National Council for Scientific and Technological Development), CAPES (Coordination for the Improvement of Higher Education Personnel), FAPEMIG (Foundation for Research Support of the State of Minas Gerais), and Pontifical Catholic University of Minas Gerais, Brazil.

\section{REFERENCES}

Abdullah, M. Time series motif discovery: dimensions and applications. WIREs Data Mining Knowl Discov vol. 4, pp. 152-159, 2014.

Caires, S. Extreme value analysis: wave data. Tech. Rep. 57, JCOMM Technical Report 57, 2011.

Chavez-Demoulin, V. and Davison, A. C. Modelling the time series extremes. Revstat Statistical Journal vol. 10, pp. 109-133, 03, 2012.

Hotelling, H. The generalization of student's ratio. In Breakthroughs in Statistics: Foundations and Basic Theory, S. Kotz and N. L. Johnson (Eds.). Springer New York, New York, NY, pp. 54-65, 1992.

Huang, D., Кoh, Y. S., And Dobbie, G. Rare pattern mining on data streams. In Proceedings of the 14th International Conference on Data Warehousing and Knowledge Discovery (DaWaK). Springer Berlin Heidelberg, Berlin, Heidelberg, pp. 303-314, 2012.

Lee, T., Zhang, R., Xiao, Y., and Dean, J. Feature extraction methods for time series data in sas enterprise miner. Tech. rep., SAS Institute, 2014.

Nakkeeran, K., Garla, S., and Chakraborty, G. Application of time series clustering using sas enterprise miner for a retail chain. Tech. rep., SAS Global Forum 2012. 04, 2012.

Ranjan, C., Reddy, M., Mustonen, M., Paynabar, K., and Pourak, K. Dataset: Rare event classification in multivariate time series. ArXiv vol. abs/1809.10717, pp. 1-7, 2018.

Serra, A. P. and ZÁrate, L. E. Characterization of time series for analyzing of the evolution of time series clusters. Expert Systems with Applications 42 (1): 596 - 611, 2015.

Taleb, N. N. The Black Swan: The Impact of the Highly Improbable. Incerto. Random House Publishing Group, London, 2007.

Trovero, M. A. and Leonard, M. J. Time series feature extraction. Tech. rep., SAS 2020-2018, 2018.

Winters, P. R. Forecasting sales by exponentially weighted moving averages. Manag. Science 6 (3): 324-342, 1960.

Yanfei Kang, Rob J Hyndman, F. L. Efficient generation of timeseries with diverse and controllable characteristics. Tech. Rep. 15/18, Monash Business School, 2018. 